\title{
É o Ronco Secundário a uma Miopatia Focal?
}

José Osmar Cardeal*

Acary Souza Bulle Oliveira**

Alberto Alain Gabbai***

\section{RESUMO}

Embora o ronco seja um distúrbio comum, sua fisiopatologia não é apropriadamente entendida. Ele poderia ser secundário ao comprometimento do controle neurológico-anatômico, ao comprometimento das vias aéreas superiores ou ambos. São apresentadas considerações segundo as quais o ronco pode estar relacionado às alterações musculares focais.

\section{UNITERMOS}

Ronco, fisiopatologia, miopatia.
* Professor-adjunto da Disciplina de Neurologia da Escola Paulista de Medicina Unifesp.

** Chefe do Setor de Doenças Neuromusculares da Disciplina de Neurologia da Escola Paulista de Medicina - Unifesp.

*** Professor Titular e Chefe da Disciplina de Neurologia da Escola Paulista de Medicina - Unifesp.

\section{INTRODUÇÃO}

Conforme estudos epidemiológicos de Lugaresi et al. ${ }^{1}$, roncadores habituais (indivíduos que roncam toda ou quase toda a noite de sono) representam $19 \%$ dos indivíduos em uma população não selecionada. Esses autores observaram que o ronco habitual é mais comum nos homens $(25 \%)$ que nas mulheres $(15 \%)$ e a freqüência aumenta com a idade após os 35 anos, em ambos os sexos, e após os 65 anos diminui lentamente nos homens; aproximadamente $60 \%$ dos homens e $40 \%$ das mulheres são roncadores habituais na faixa etária entre 41 e 65 anos. Resultados semelhantes foram observados por outros autores. Assim, Koskenvuo et $a l .^{2}$ observaram que $30 \%$ dos indivíduos com idade entre 40 e 69 anos são roncadores habituais, enquanto Gislason et al. ${ }^{3}$ observaram $15,5 \%$ entre 30 e 69 anos de idade.

A obesidade aumenta a freqüência do ronco e da síndrome da apnéia obstrutiva do sono (SAOS), conforme tem sido registrado nos estudos epidemiológicos. Ronco habitual foi observado em 2,8\% das mulheres e $7 \%$ dos homens com índice de massa corpórea (IMC) abaixo de $27 \mathrm{~kg} / \mathrm{m}^{2} \mathrm{e}$, acima desses níveis, essa freqüência sobe para $13,9 \%$ e $61 \%$, respectivamente $^{2}$. Em um grupo de 41 adolescentes com peso superior a $150 \%$ do ideal, o ronco ocorreu em todos ${ }^{4}$. Por outro lado, em $75 \%$ dos pacientes com SAOS foi observado IMC superior a $20 \%$ do ideal ${ }^{5}$.

O ronco é um pré-requisito para a síndrome de apnéia do sono, e estudos recentes enfatizam que essa síndrome tenha uma predisposição familiar ${ }^{6}$. Lugaresi et al. ${ }^{7}$ sugerem que, havendo predisposição genética para o ronco e para a SAOS, essa predisposição poderia ser anatômica (estreitamento congênito das fauces, por exemplo), funcional (por exemplo, defeito de coordenação da atividade dos músculos dilatadores da faringe e do diafragma) ou constitucional (por exemplo, hábito pletórico, pescoço curto, obesidade). De modo geral, existem três hipóteses para atuação desses fatores genéticos nos distúrbios respiratórios do sono (DRS). A primeira considera que vias aéreas de menores dimensões ocorreriam em familiares de indivíduos com DRS e genes controladores do desenvolvimento craniofacial poderiam predispor a alterações maxilomandibulares e faciais. A segunda considera que genes controladores do desenvolvimento da quimiossensibilidade 
determinariam disfunções ventilatórias. Uma terceira considera a possibilidade de interação genética envolvendo fatores ventilatórios e anormalidades anatômicas $8,9,10,11$.

\section{FISIOPATOLOGIA DO RONCO}

Alguns eventos relevantes são encontrados em revisão sobre os mecanismos fisiopatológicos do ronco, tais como: a capacidade funcional residual pulmonar e a complacência pulmonar são reduzidas durante o sono; o aumento da pressão intratorácica negativa poderia resultar em colapso das paredes da orofaringe; o ronco e a apnéia obstrutiva resultam de estreitamento ou completa oclusão da orofaringe aos efeitos do sono ${ }^{7}$. O estreitamento das vias aéreas superiores (VAS) tem sido motivo de algumas especulações. A deposição local de gordura tem sido considerada ${ }^{12}$, embora nem sempre o paciente obeso apresente comprometimento das VAS ${ }^{13}$. Defeitos nos mecanismos de controle respiratório ${ }^{14} \mathrm{e}$ da quimiossensibilidade ${ }^{15}$ têm sido observados. Até o momento, não sabemos se são distúrbios funcionais primários ou determinados por outros fatores.

Uma condição anatômica freqüente, avaliada em pacientes com ronco habitual e em pacientes com ronco associado à apnéia, é a obstrução nasal, sendo sugerido que sua correção possa melhorar o ronco ${ }^{16}$, mas não há evidências de eficácia para tratar eventos respiratórios do sono.

A avaliação da morfologia craniofacial, com estudos cefalométricos diversificados, analisando estruturas ósseas e partes moles como a língua, o palato mole e a faringe, tem procurado encontrar parâmetros preditivos para o ronco e para a SAOS. Até o momento, não se sabe a relevância de anormalidades encontradas a partir desses estudos anatômicos. Por outro lado, há indícios de que anormalidades encontradas em estudos cefalométricos, particularmente em tecidos moles, ocorram mais provavelmente como conseqüência do ronco habitual e da SAOS do que de um evento primário ${ }^{17}$. Assim, em estudos recentes, a instabilidade das VAS e seu controle muscular parecem fatores mais relevantes na fisiopatologia dos DRS do que puros estreitamentos anatômicos ${ }^{18}$.

Durante o sono, uma leve redução do fluxo aéreo inspiratório está associada com o ronco e uma redução mais intensa, com apnéias e hipopnéias obstrutivas. Foi observado que essa redução do fluxo aéreo, avaliado por medidas pressóricas, associa-se com o colapso das vias aéreas em um continuum desde os assintomáticos, passando pelos roncadores habituais, até os apnéicos ${ }^{19}$.
Estudos mais recentes demonstram que essa redução do fluxo aéreo, em roncadores habituais e em apnéicos, não se restringe ao fluxo inspiratório, mas que o processo de obstrução das VAS é evento inspiratório e expiratório ${ }^{20}$. Redução do fluxo em roncadores habituais e em casos de apnéias obstrutivas foi igualmente observada, durante o sono, em estudos obtidos de imagens cinerradiográficas, indicando ser evento cuja presença é obrigatória para a produção do ronco, o qual parece decorrer de vibrações do palato mole, paredes faríngeas, epiglote e língua ${ }^{21}$.

Tem-se procurado identificar o local ou os locais envolvidos na obstrução das vias aéreas superiores em pacientes com DRS. Um estudo feito com nasoendoscopia em sono, obtida de 50 pacientes adultos com ronco, sem apnéias, mostrou que em $70 \%$ deles o ronco era produzido por flutter palatal isolado e em $20 \%$ havia flutter palatal associado com outros locais de produção do ruído. Em $10 \%$, o ronco era produzido na região supraglótica, em $8 \%$, na tonsilar e, em $2 \%$, na base da língua ${ }^{22}$. Com o auxílio de 5 transdutores de pressão, Skatvedt ${ }^{23}$ analisou pacientes com SAOS e observou que, dentre os 20 pacientes que apresentavam obstrução durante o sono, 7 tiveram obstrução em apenas um segmento, 9, em dois e 4, em 3 segmentos. Nesse mesmo estudo, o autor avaliou, ainda, 10 pacientes, 3 meses após tratamento cirúrgico por uvalopalatofaringoplastia (UPPP) e notou que apenas 4 ficaram livres de obstrução, enquanto 6 ainda apresentavam obstrução envolvendo os segmentos velofaríngeos.

Em outro estudo ${ }^{24}$, feito pela análise de imagens obtidas com ressonância magnética das vias aéreas superiores de 21 indivíduos normais, 21 roncadores com poucas apnéias e 26 com apnéias obstrutivas freqüentes, chegou-se aos seguintes resultados:

1) o segmento com área mínima foi significativamente menor nos apnéicos comparados com os normais e ocorreu na região retropalatal;

2) o estreitamento da via aérea nos apnéicos predominou nas dimensões laterais, sem alteração significativa na medida ântero-posterior;

3) a distância entre os ramos da mandíbula não foi diferente entre os grupos;

4) o estreitamento da parede lateral foi devido predominantemente à espessura da parede faríngea, não relacionado com deposição de gordura;

5) o tamanho do coxim adiposo, no segmento com mínima área nas VAS, não era maior nos apnéicos que nos normais.

Gleadhill et al. ${ }^{19}$ verificaram que a composição histológica do músculo constritor médio da faringe 
(MCMF) dos indivíduos não-roncadores é muito semelhante à dos músculos de membros, exceto por serem de menor diâmetro e por apresentarem menor proporção de fibras musculares IIb, com subseqüente maior proporção de fibras I e IIa. Esses autores notaram que o MCMF de roncadores habituais apresenta anormalidade na composição de suas fibras musculares faríngeas quando comparado com indivíduos normais. Essa composição anormal caracteriza-se por hipertrofia e preponderância ainda maior de fibras IIa. Nesse estudo, não foram observadas alterações miopáticas ou neurogênicas. Para explicar os achados dessa pesquisa, os autores postularam dois mecanismos:

1) a redução constitucional dos neurônios motores alfa lentos induz uma transformação adaptativa das fibras IIb, com subseqüente aumento em número e hipertrofia das fibras IIa;

2) os motoneurônios alteram seus padrões de descargas, modificando a distribuição do tipo de fibras do MCMF como uma adaptação para as características das VAS dos roncadores habituais.

Outro estudo histológico, feito por Woodson et al. ${ }^{25}$, com secções transversas do palato mole distal e da úvula de três grupos de indivíduos, com 4 em cada um deles, sendo um formado por roncadores habituais com índice de apnéia menor do que 20 apnéias/h, um grupo com mais de 50 apnéias/h e outro por não-roncadores, mostrou que tanto nos roncadores habituais como nos apnéicos havia hipertrofia de glândulas mucosas com dilatação de seus ductos e metaplasia escamosa, fibras musculares fendidas por infiltração de glândulas mucosas, atrofia focal de fibras musculares e extenso edema da lâmina própria com dilatação vascular. Essas alterações qualitativas ocorreram tanto nos roncadores habituais como nos com SAOS, embora com menor intensidade em alguns roncadores habituais. Nenhum dos achados associou-se a desenvolvimento de apnéia obstrutiva. Nesse estudo, achados adicionais foram obtidos com a microscopia eletrônica e eram caracterizados por freqüente degeneração focal de fibras e de axônios mielinizados. Segundo os autores, as anormalidades histológicas, semelhantes em roncadores habituais e apnéicos, são favoráveis a postulações anteriores de uma etiologia comum para o ronco habitual e para a SAOS, contrárias à idéia de apnéia decorrente de trauma vibratório faríngeo produzido pelo ronco. Adicionalmente, argumentaram que alterações em nervos periféricos, observadas na microscopia eletrônica, podem contribuir para a instabilidade das VAS e para o desenvolvimento da apnéia obstrutiva por comprometimento dos reflexos faríngeos.
Outra evidência de que as anormalidades musculares, incluindo lesões neurogênicas, podem estar relacionadas com o ronco e o desenvolvimento de apnéia obstrutiva do sono foi recentemente obtida por Friberg et al. ${ }^{26} \mathrm{em}$ estudos feitos a partir de biópsias de músculo palatofaríngeo (MP) em 21 roncadores habituais, em $10 \mathrm{com}$ diferentes índices de apnéia obstrutiva do sono e comparado com grupo de 10 indivíduos-controle normais. Foram observadas fibras musculares hipertrofiadas ou atrofiadas nos pacientes roncadores e nos pacientes com SAOS, freqüentemente com alteração no padrão de distribuição das fibras (type grouping), sugerindo comprometimento neurogênico. As anormalidades correlacionaram-se de modo significativo com o grau de DRS. Tendo o músculo palatofaríngeo grande importância na faringe, a sua exposição a trauma freqüente, produzido por vibração de baixa freqüência decorrente do ronco, ocasionaria comprometimento do nervo periférico sob forma permanente e alteração de função.

Sériès et al. ${ }^{27}$ estudaram características da fibra muscular e de seu metabolismo em dois diferentes músculos das vias aéreas superiores - músculo genioglosso (MG) e músculo da úvula (MU) - obtidos durante uvulopalatoplastia de pacientes roncadores e de pacientes com SAOS. Foi observado que o MU apresenta atividade enzimática glicolítica, glicogenolítica e anaeróbia significativamente maior nos pacientes com SAOS que nos roncadores. Havia uma maior proporção de fibras IIa (fibras Ia: fibras aeróbicas, com alto poder oxidativo, com função de contração lenta e de resistência; fibras IIb: fibras anaeróbicas, com alto poder glicolítico, com função de contração rápida e de força; fibras IIa: fibras intermediárias, com atividade oxidativa e glicolítica). Já a proporção de fibras aeróbicas era maior no $\mathrm{MG}$ que no $\mathrm{MU}$, mas não se encontraram diferenças entre pacientes com SAO e os apenas roncadores.

A teoria neurogênica para explicar o colapso das VAS em pacientes com ronco tem sido testada em alguns estudos funcionais. Friberg et al. ${ }^{28}$, usando uma técnica de perfusão por laser doppler, combinada com estimulação elétrica para investigar a regulação da aferência nervosa da microcirculação do palato mole, avaliaram pacientes com ronco e pacientes com SAOS, comparando-os com grupo-controle de indivíduos normais. Foi observado que a estimulação elétrica determina vasodilatação exagerada nos pacientes roncadores e nos com grau leve de apnéia obstrutiva quando comparados aos normais. Em contraste, a vasodilatação produzida pela estimulação elétrica foi significativamente reduzida nos pacientes com grau 
intenso de apnéia obstrutiva comparados com os normais. Esses resultados foram interpretados como suporte para a hipótese de lesão progressiva da inervação aferente local nos pacientes roncadores habituais com ou sem apnéia. Uma explicação para o aumento da vasodilatação seria a presença de brotamento (sprouting) intraepitelial e/ou subepitelial, pois é sabido que sprouting é um achado bem conhecido de lesão de nervo aferente.

Um outro estudo recente, feito por Friberg et al. ${ }^{29}$, admitindo o ronco habitual preceder a SAOS e que a patência das VAS depende de mecanismo reflexo que atua na vigência de pressão intrafaríngea negativa, possivelmente mediada por receptores localizados na mucosa faríngea, ou seja, terminações nervosas aferentes, procuraram elucidar os mecanismos fisiopatológicos subjacentes à essa progressão. Tais nervos contêm uma proteína específica do nervo, pretein-gene product 9.5 (PGP 9.5), e, em alguns casos, substância $\mathrm{P}$ (SP) e calcitonina gene-related (CGRP). O material utilizado foi obtido por biópsias da mucosa do palato mole, de homens não-fumantes, 10 com SAOS, 11 nãoroncadores-controle, e os principais resultados desse estudo imunoistoquímico foram:

1) 9 dentre 10 pacientes com SAOS e 4 dentre os 11 roncadores habituais apresentaram aumento do número de nervos PGP-, SP- e CGRP-imunorreativos;

2) aumento de densidade de terminações nervosas varicosas na papila e no epitélio do palato mole. Esses achados foram interpretados pelos autores por serem indicativos de lesão de nervos aferentes e por suportarem a hipótese de lesão neurogênica progressiva como um fator que contribui para o colapso das vias aéreas durante a SAO no sono.

\section{COMENTÁRIOS}

Esses trabalhos são indicativos de que alterações morfológicas nos músculos das vias aéreas superiores têm uma relação íntima com a manutenção do ronco e com a SAOS.

O músculo esquelético adapta-se tanto em seus aspectos metabólicos como histoquímicos às características de força que ele deve produzir. Músculos que necessitam de atividade contínua (endurance) exercícios de longa duração, com contração lenta exibem uma maior proporção de fibras aeróbicas (tipo I) e maior densidade mitocondrial. Por outro lado, músculos adaptados para exercícios máximos e de menor duração necessitam de fibras constituídas para produzir contrações rápidas, equipadas com atividade enzimática anaeróbica elevada (atividade glicolítica) para produção de força máxima.

Sabe-se que a atividade contrátil constantemente elevada modifica a distribuição das fibras musculares em um processo adaptativo para as diferentes necessidades funcionais. O treino de resistência (endurance) pode alterar a composição das fibras tipo II, com aumento das fibras IIa e diminuição subseqüente das fibras IIb.

As diferenças histoquímicas do músculo da úvula entre pacientes com SAOS e os apenas roncadores podem ser interpretadas como conseqüência de um processo adaptativo desde que a atividade contrátil intermitente máxima seja relacionada com o quadro de SAOS. A atividade muscular contínua (over use) poderia lesar o botão nervoso terminal e o processo de reinervação pelo brotamento (sprouting) justificaria o encontro de vasodilatação exagerada nos pacientes roncadores e nos portadores de apnéia obstrutiva quando submetidos à estimulação elétrica.

Assim, os estudos sobre a fisiopatologia do ronco têm proporcionado relevantes conhecimentos, os quais poderão abrir novas perspectivas para a busca de melhores resultados em tratamentos de pacientes com ronco e daqueles com ronco e SAOS.

\section{SUMMARY}

Is the Snoring Related to Focal Myopathy?

The pathophysiologic events that cause snoring are poorly understood. Snoring may be secondary to changes in neurologic control, airway flow, or both. In this review we speculate that besides the previous mentioned factors, snoring may be caused by a focal myopathy.

\section{KEYWORDS}

Snoring, pathophysiology, myopathy.

\section{Referências}

1. Lugaresi E, Cirignotta F, Coccagna G, Piana C. Some epidemiological data on snoring and cardiocirculatory disturbances. Sleep, 3:221-224, 1980.

2. Koskenvuo M, Kaprio J, Partnen $\mathrm{M}$ et al. Snoring as a risk factor for hypertension and angina pectoris. Lancet, i:893896, 1985.

3. Gislason $\mathrm{T}$, Aberg $\mathrm{H}$, Taube A. Snoring and systemic hypertension: an epidemiological study. Acta Med Scand, 222:415-421, 1987.

4. Mallory GB Jr, Fiser DH, Jackson R. Sleep-associated breathing disorders in morbidly obese children and adolescents. J Pediatr, 115:892-7, 1989.

5. Walsh RE, Michaelson ED. Upper airway obstruction in obese patients with sleep disturbance and somnolence. Ann Intern Med, 76:185-192, 1972. 
6. Pillar G \& Lavie P. Assessment of the role of inheritance in sleep apnea syndrome. Am J Respir Crit Care Med, 151(3 Pt 1):688-91, 1995.

7. Lugaresi E, Cirignotta F, Montagna P, Sforza E. Snoring: pathogenic, clinical, and therapeutic aspects. In: Kryger $\mathrm{MH}$, Roth T \& Dement WC (eds.). Principles and practice of sleep medicine. W.B. Saunders Co., London, 1994, cap. 61, 621$629 \mathrm{pp}$.

8. Guilleminault C, Heldt G, Powell N, Riley R. Small upper airway in near-miss sudden infant death syndrome infants and their families. Lancet, i:402-407, 1986.

9. Arkinstall WW, Nirmel K, Klissouras V, Milic-Emili J. Genetic differences in the ventilatory response to inhaled CO2. J Appl Physiol, 36:6-11, 1974.

10. Kawakami Y, Yoshikawa T, Shida A, Asanuma Y, Murao M. Control of breathing in young twins. J Appl Physiol, 52:537542, 1982.

11. el Bayadi S, Millman RP, Tishler PV, Rosenberg C, Saliski W, Boucher MA, Redline S. A family study of sleep apnea. Anatomic and physiologic interactions. Chest, 98:554-9, 1990.

12. Lowe AA, Fleetham JA, Adachi S, Ryan CF. Cephalometric and computer tomographic predictors of obstructive sleep apnea severity. Am J Orthod Dendofacial Orthop, 107:58995, 1995.

13. Guilleminault C, Partinen M, Hollman K, Powell N, Stoohs R. Familial aggregates in obstructive sleep apnea syndrome. Chest, 107:1545-51, 1995.

14. Deegan PC, McNicholas WT. Pathophysiology of obstructive sleep apnea. Eur Resp J, 8:1161-78, 1995.

15. Gold AR, Schwartz AR, Wise RA, Smith PL. Pulmonary function and respiratory chemosensitivity in moderately obese patients with sleep apnea. Chest, 103:1325-9, 1993.

16. Low WK. Can snoring relief after nasal septal surgery be predicted? Clin Otolaryngol, 19:142-4, 1994.

17. Zucconi M, Ferini Strambi L, Palazzi S, Orena C, Zonta S, Smirne S. Habitual snoring with and without obstructive sleep apnea: the importance of cephalometric variables. Thorax, 47:157-61, 1992 .

18. Boot $\mathrm{H}$, van der Meche FG, Poublon RM, Bogaard JM, Gainai AZ, Schmitz PI. Upper airway patency and nocturnal desaturation in habitual snoring and obstructive sleep apnea: pathogenesis of sleep-related breathing disorders. Eur Neurol, 36:206-10, 1996.

19. Gleadhill IC, Schwartz AR, Schubert N, Wise RA, Permutt $\mathrm{S}$, Smith PL. Upper airway collapsibility in snorers and in patients with obstructive hypopnea and apnea. Am Rev Respir Dis, 143:1300-3, 1991.
20. Stanescu D, Kostianev S, Sanna A, Liistro G, Veriter C. Expiratory flow limitation during sleep in heavy snorers and obstructive sleep apnea patients. Eur Respir J, 9:2116-21, 1996.

21. Liistro G, Stanescu DC, Veriter C, Rodenstein DO, Aubert Tulkens G. Pattern of snoring in obstructive sleep apnea patients and in heavy snorers. Sleep, 14:517-25, 1991.

22. Quinn SJ, Daly N, Ellis PD. Observation of the mechanism of snoring using sleep asendoscopy. Clin Otolaryngol, 20:360-4, 1995.

23. Skatvedt $O$. Continuous pressure measurements during sleep to localize obstructions in the upper airways in heavy snorers and patients with obstructive sleep apnea syndrome. Eur Arch Otorhinolaryngol, 252:11-4, 1995.

24. Schwab RJ, Gupta KB, Gefter WB, Metzger LJ, Hoffman EA Pack Al. Upper airway and soft tissue anatomy in normal subjects and patients with sleep-disordered breathing. Significance of the lateral pharyngeal walls. Am J Respir Crit Care Med, 152(5 Pt 1):1673-89, 1995.

25. Woodson BT, Garancis JC, Toohill RJ. Histopathologic changes in snoring and obstructive sleep apnea syndrome. Laryngoscope, 101(12 Pt 1):1318-22, 1991.

26. Friberg D, Ansved T, Borg K, Carlsson Nordlander B, Larsson $\mathrm{H}$, Svanborg E. Histological indications of a progressive snorers disease in an upper airway muscle. Am J Respir Crit Care Med, 157:586-93, 1998.

27. Sériès FJ, Simoneau SA, St Pierre S, Marc I. Characteristics of the genioglossus and musculus uvulae in sleep apnea hypopnea syndrome and in snorers. Am J Respir Crit Care Med, 153(6 Pt 1):1870-4, 1996.

28. Friberg D, Gazelius B, Lindblad LE, Nordlander B. Habitual snorers and sleep apnoics have abnormal vascular reactions of the soft palatal mucosa on afferent nerve stimulation. Laryngoscope, 108:431-6, 1998.

29. Friberg D, Gazelius B, Hökfelt T, Nordlander B. Abnorma afferent nerve endings in the soft palatal mucosa of sleep apnoics and habitual snorers. Regul Pept, 71:29-36, 1997.

Endereço para correspondência:

José Osmar Cardeal

Rua Borges Lagoa, 873 - cj. 11

CEP 04038-031 - São Paulo, SP

E-mail: cardeal@sun-nepi.epm.br 